\title{
ON THE CONSTRUCTION OF A SAFETY GAP PREDICTION MODEL FOR FREEWAY BUS LANE-CHANGING MANEUVER USING DRIVING SIMULATOR DATA
}

\author{
Chao-Hua Chen ${ }^{1}$ and Wen-Chu $\mathrm{Ho}^{2}$ \\ ${ }^{1}$ Department of Transportation Technology and Logistics Management \\ ${ }^{2}$ Institute of Technology Management \\ Chung Hua University \\ Hsinchu, TAIWAN \\ E-mail: erchen@chu.edu.tw
}

\begin{abstract}
Summary: Lane-change crashes are not only responsible for an important portion of vehicular fatalities, but also for crash-caused traffic delays, often resulting in congestion. The type of discretionary lane change was the focus of this research, in which a safety gap prediction model was constructed for potential application in the development of lane-change support systems. Data for analysis and model fitting were collected from a fixed-based bus driving simulator. The experimental scene designed for the driving simulator consisted of a straight section of two-lane freeway mainline with daylight and vehicular flows traveling at different speed levels on the road. Ten professional coach drivers were recruited to perform lane-change experiments. Results of two-way ANOVA revealed a significant lane-change direction $\times$ vehicle speed on the target lane interaction, and further analyses demonstrated that there was a simple effect for vehicle speed on the target lane in the left-to-right group of the type of lane-change direction factor. A safety gap forecasting model with the time gap between lead and lag vehicle on the target lane as the forecasted variable was constructed, and tests of true out-of-sample forecast accuracy of the prediction model showed promising results for its potential application in the development of lane-change support systems.
\end{abstract}

\section{INTRODUCTION}

National Highway Traffic Safety Administration (NHTSA) research (2004) indicated that lane-change crashes account for $4 \%$ to $10 \%$ of all crashes, and between 0.5 to $1.5 \%$ of all motor vehicle fatalities per year. Furthermore, other NHTSA research (1994) pointed out that one apparent reason for lane-change crashes was drivers' not seeing or being unaware of the other vehicle when making lane changes. Therefore, it is expected that lane-change crashes could be prevented - or at least collision risk might be decreased - by providing aids to drivers.

Research on vehicle lane change has been a popular topic in literature, where the major focus has been on the study of lane-change behavior and lane-change decision structure. One primary application for such research is in traffic simulation, where the concept of accepted gap for lane change was proposed to develop lane-change models and used in conjunction with the application of probabilistic choice models to construct lane-changing decision structures (Mahmassani \& Sheffi, 1981). In 1986, Gipps proposed a general lane-change decision model for cars and trucks driving in urban streets, which could be summarized into three major steps: (1) the motivation to make a lane change, (2) selecting the target lane for lane-changing, and (3) accepting a safety gap and performing the lane change. Many traffic-flow simulation models applied Gipps' lane-change decision model to simulate vehicle lane-change maneuvers. In CORSIM (FHWA, 1998) and some 
major research (Ahmed, Ben-Akiva, Koutsopoulos, \& Mishalani, 1996; Toledo, Koutsopoulos, \& Ben-Akiva, 2003) the types of lane change were classified as either mandatory or discretionary. Mandatory lane change logic was applied to a driver when the driver was required to leave the current lane; discretionary lane change logic was used when the driver perceived the driving conditions in the target lane to be better, although a lane change was not required. Another type of research (Jula, Kosmatopoulos, \& Ioannou, 2000; Winsum, Waard, \& Brookhuis, 1999; NHTSA, 2004) focused on the development of a lane-change module for application in the development of advanced safety vehicle or the development of collision avoidance warning systems. The subject of these studies was also cars, and they focused more on the research of vehicle kinemics involved in lane-change maneuvers for finding the machinery and physical properties that enable safe lane-change maneuvers. In addition, these studies generally adopted the critical gap as the necessary condition for control of safe lane-change maneuvers.

In the aforementioned reviews, the size of the accepted gap on the target lane was recognized as a critical factor affecting whether a driver was able to execute a safe lane change. The critical gap was generally defined as the minimum value of the accepted gap. Furthermore, past research (Ahmed et al., 1996; Toledo et al., 2003) indicated that the size of the accepted gap could vary with the speed of the subject vehicle, its lead and lag vehicle, the longitudinal inter-vehicle' spacing, traffic conditions, and driver characteristics, etc. Therefore, the concept of accepted gap and critical gap were adopted in this study to develop a safety gap prediction model for potential application in the development of lane-change support systems. This paper first presents the methodology, and then the demonstration of analysis results. The discussion of research results and conclusions will be drawn in the last section.

\section{METHODOLOGY}

\section{Driving Simulator and Experiment}

The bus driving simulator used as the platform for the lane-change simulation is set up in Chung Hua University, Hsinchu, Taiwan. Detailed layout of this simulator is illustrated in Figure 1. The simulator includes the front buck of a bus that has been instrumented to collect standard driver data, such as steering wheel position, throttle and brake position, turn signal, etc. Physical validation of the simulator on throttle and brake was made according to the Japanese Industrial Standard (JIS) D1014 and D1013. The simulated environment is projected from the back onto the screen surrounding the front buck of the bus. Two side-view mirrors in the buck were not used, but a simulated side-view mirror was incorporated into each side of the projected environment at the visual angle of the actual side-view mirror. The installation of the simulated side-view mirrors enabled drivers to be aware of the simulated surrounding vehicles.

The simulated driving environment developed for this study was a multi-lane freeway under daylight conditions. The scene included a straight and flat mainline section, two lanes plus one shoulder lane in each direction, standard lane markings, and a guardrail barrier off the side of the road. The traffic conditions of the simulated environment were designed with $\mathrm{C}$ level-of-service vehicular flows (equivalently to 18 to 23 vehicles per $\mathrm{km}$ per lane and speeds greater than $80 \mathrm{~km} / \mathrm{hr}$ ) traveling in the lane on which the subject vehicle traveled, and vehicular flows with three different speed levels, 90,100 , or $110 \mathrm{~km} / \mathrm{hr}$, traveling in the subject vehicle's neighboring lane, but with 
less flow density. The idea behind setting higher speed levels on the target lane was to create a driving environment that would easily trigger the subject vehicle to make discretionary lane changes when desired. Simulated vehicles in the target lane were moved by platoons, with non-cooperative mechanism among vehicles and discharged at constant speeds. Two types of platoons were designed with different combinations of inter-vehicle gaps. One type consisted of gaps randomly selected by the simulator and the other contained gaps permutated from small to large sizes at a unit of 0.1 meters. The sizes for both types of gaps were within the pre-designed ranges. The ranges of gaps for 90,100 and $110 \mathrm{~km} / \mathrm{hr}$ speed levels were set as 30-37, 33-39 and 34-41 meters, respectively, by referencing the research results of Ahmed et al. (1996) regarding the investigation of the lead and lag gap of drivers when making mandatory lane changes.
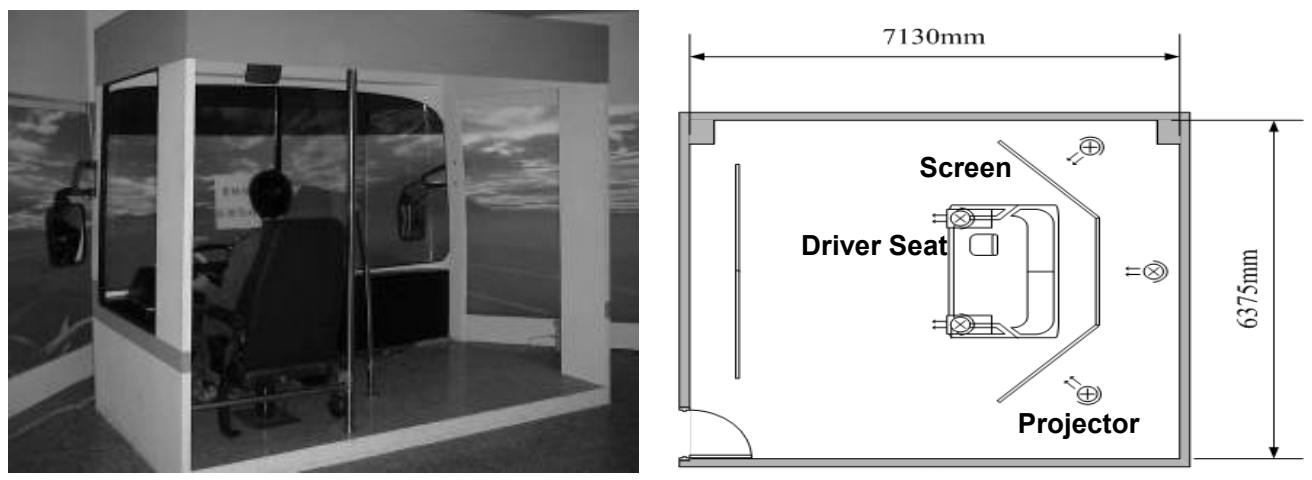

Figure 1. The Fixed-base Bus Driving Simulator

The simulation experiment for this study was designed in two stages, where the first stage was for drivers to practice and the second stage was the formal experiment. Before starting the experiment, subjects were educated to follow one rule, which was to signalize the turn-signal prior to executing the lane-change maneuver. At the end of the first stage, we also collected the subject's viewpoints regarding the factuality of the system through a questionnaire survey for the purpose of system improvement. During the second stage, the simulation procedure began with the subject vehicle starting the engine and accelerating to the desired speed, which was above $85 \mathrm{~km} / \mathrm{hr}$ and below the speed limit of $110 \mathrm{~km} / \mathrm{hr}$. After the subject vehicle had followed the front vehicle for 10 seconds, simulated vehicles were discharged onto the neighboring lane, whose speed levels and types of platoons were randomly selected by the simulator. The system then activated the front vehicle to decelerate from $100 \mathrm{~km} / \mathrm{hr}$ to $85 \mathrm{~km} / \mathrm{hr}$ to trigger the subject vehicle to make a discretionary lane change. After the subject vehicle completed the lane-change maneuver and followed the new front vehicle for 10 seconds, the system again activated the front vehicle to decrease to $85 \mathrm{~km} / \mathrm{hr}$ to trigger the subject vehicle to make a lane change. The total simulation time for the formal experiment was 30 minutes, with a break every 15 minutes. For each 15 minutes of simulation, one type of platoon would be selected randomly without repetition, and in each 5 minutes, one speed level would be randomly selected from the preset speed levels without repetition for simulating neighboring traffic.

Experimental subjects were professional coach drivers. Ten drivers were recruited from several private transportation companies in Taiwan. All of the drivers were male, with at least eleven years of driving experience and between 25 and 60 years of age. 


\section{Data Extraction and Analysis}

The simulator, in conjunction with the driving environment, produced a continual stream of data, which were recorded every 0.03125 seconds. Information recorded by the simulator is summarized in Table 1. Due to the fact that the successful lane-change data had to be extracted from the recorded continuous data, we decided the time-point for data extraction would be the time the subject vehicle signalized the turn-signal, since this signal is the only sign that can be used to confirm the subjection vehicle's lane-change action. Relative data were extracted immediately after this time-point to compute the values of lane-change variables determined for constructing a safety gap prediction model. The preset lane-change variables for analysis and construction of a prediction model are presented in Table 2. Figure 2 illustrates the definition of each gap under lane-change maneuvers.

Table 1. Recording Information of the Driving Simulator

\begin{tabular}{|l|l|}
\hline Subject & \multicolumn{1}{c|}{ Information } \\
\hline $\begin{array}{l}\text { Subject } \\
\text { vehicle }\end{array}$ & $\begin{array}{l}\text { Driver data (subject number, system time, steering wheel angle, throttle position, brake } \\
\text { position, acceleration, turn signal, x-coordinate, y-coordinate, velocity, angle velocity); } \\
\text { Crash data (recorded as 1 for safe and 0 for crash). }\end{array}$ \\
\hline $\begin{array}{l}\text { Surrounding } \\
\text { traffic of the } \\
\text { subject vehicle }\end{array}$ & $\begin{array}{l}\text { Gap data (the corresponding gap measure on the target lane); Front vehicle data (code } \\
\text { number, y-coordinate and velocity of the front vehicle); Rear vehicle data (code number } \\
\text { and y-coordinate of the rear vehicle); Neighboring lead vehicle data (code number and } \\
\text { y-coordinate of the front vehicle on the target lane); Neighboring lag vehicle data (code } \\
\text { number and y-coordinate of the rear vehicle on the target lane); Velocity data on the } \\
\text { target lane (vehicle velocity on the target lane). }\end{array}$ \\
\hline
\end{tabular}

Table 2. Lane-change Variables for Investigation and Model Construction

\begin{tabular}{|c|l|}
\hline Variable & \\
\hline$V_{s}$ & Speed of the subject vehicle \\
\hline$V_{f}$ & Speed of the subject vehicle's front vehicle \\
\hline$d V_{f}$ & Speed difference of $\mathrm{V}_{\mathrm{f}}$ and $\mathrm{V}_{\mathrm{s}}\left(\mathrm{dV}_{\mathrm{f}}=\mathrm{V}_{\mathrm{f}}-\mathrm{V}_{\mathrm{s}}\right)$ \\
\hline$G_{f}$ & Gap between the subject vehicle and its front vehicle \\
\hline$V_{n l}$ & Speed of the vehicle on the target lane \\
\hline$d V_{n l}$ & Speed difference of $\mathrm{V}_{\mathrm{nl}}$ and $\mathrm{V}_{\mathrm{s}}\left(\mathrm{d} \mathrm{V}_{\mathrm{nl}}=\mathrm{V}_{\mathrm{nl}}-\mathrm{V}_{\mathrm{s}}\right)$ \\
\hline$G_{n l}$ lead & Gap between the subject vehicle and its lead vehicle on the target lane \\
\hline$G_{n l}$ lag & Gap between the subject vehicle and its lag vehicle on the target lane \\
\hline$G$ & The accepted gap in the target lane \\
\hline
\end{tabular}

The lane-change sampling data were also examined before they were used in analysis. Some of the samples that were generated by drivers not following the discretionary lane-change condition set in the experiment were further excluded from analysis. More specifically, only the samples for condition-satisfied and successful discretionary lane change were considered for analysis. The information about the lane-change samples used in analysis is depicted in Table 3, where unbalanced group samples were revealed. Additionally, the mean size of the accepted gap for the left-to-right and right-to-left lane changes was 34.31 and 33.61 meters, respectively. 


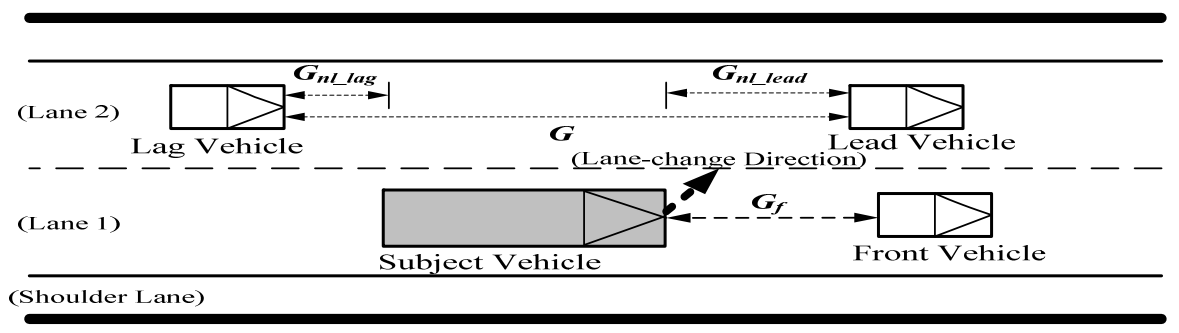

Figure 2. Illustration of Lane-change Variables

Table 3. Samples for Analysis

\begin{tabular}{|l|c|c|c|c|}
\hline \multirow{2}{*}{ Type of Lane-change } & \multicolumn{2}{|c|}{ Vehicle speed on the target lane $(\mathrm{km} / \mathrm{hr})$} & \multirow{2}{*}{ Total } \\
\cline { 2 - 4 } & 90 & 100 & 110 & \\
\hline Right-to-Left & 9 & 14 & 19 & 42 \\
\hline Left-to-Right & 31 & 32 & 23 & 86 \\
\hline Total & 40 & 46 & 42 & 128 \\
\hline
\end{tabular}

\section{RESULTS}

\section{Analysis of Variance}

The research design used in this study was a factorial design with two between-group factors (lane-change direction and vehicle speed on the target lane), and one criterion variable (the accepted gap). Two-way ANOVA was used to determine the effect of each factor on the size of the accepted gap. The GLM procedure in SAS was used to tackle the problem of unbalanced group samples. Analysis results revealed a significant lane-change direction $\times$ vehicle speed on the target lane interaction, $\mathrm{F}(2,122)=3.82, \mathrm{p}<.025$, and the nature of this interaction is displayed in Figure 3. Subsequent analyses demonstrated that there was a simple effect for vehicle speed in the target lane for the left-to-right group of the type of lane-change direction factor, $F(2,83)=16.18, \mathrm{p}$ $<.0001$. As Figure 3 shows, the group with $110 \mathrm{~km} / \mathrm{hr}$ of vehicle speed in the target lane displayed larger sizes of accepted gaps than the group with $100 \mathrm{~km} / \mathrm{hr}$ of vehicle speed in the target lane, which, in turn, demonstrated larger sizes of accepted gaps than the group with $90 \mathrm{~km} / \mathrm{hr}$ of vehicle speed in the target lane. The simple effect for vehicle speed in the target lane for the right-to-left group of the type of the lane-change direction factor proved to be nonsignificant, F (2.39) $=0.52$, $\mathrm{p}>.05$.

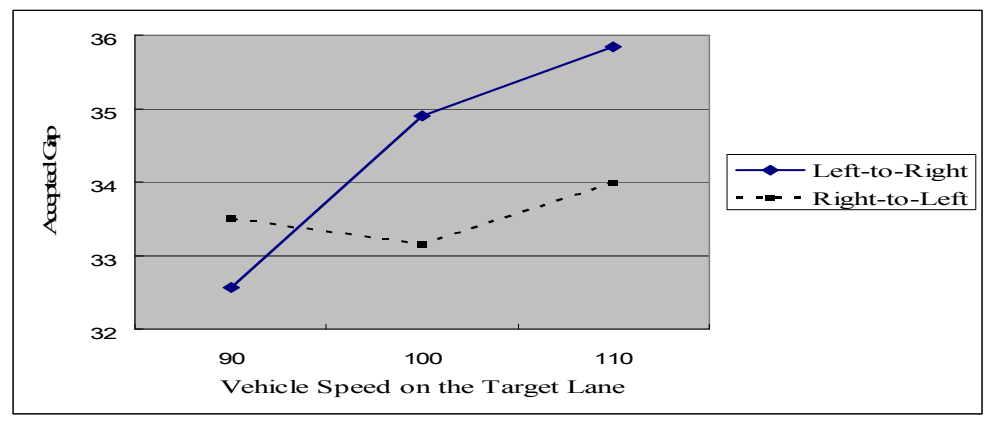

Figure 3 Mean Levels of Accepted Gap as a Function of the Interaction between Lane-change Direction and Vehicle Speed on the Target Lane 


\section{Safety Gap Prediction Model}

Multiple regression method was applied to construct a safety gap prediction model with the accepted gap as the dependent variable. Two types of accepted gaps were considered: the gap measure in distance and the gap in time. The value of a time gap, denoted as "time_Gap", is equal to the spacing of an accepted gap divided by the vehicle speed in the target lane. The independent variables adopted for model construction are those listed in Table 3. Based on the extracted lane-change samples and the use of stepwise regression method, the best-fit model is time_Gap $=\operatorname{Exp}\left(0.642-0.011 V_{n l}+0.012 G_{n l_{\text {_lead }}}+0.012 G_{n l_{\text {_lag }}}+0.004 V_{s}\right)$ with $\mathrm{R}^{2}=0.62$ and estimated parameters all being significant at $\mathrm{p}<.0005$. Residual analysis through the examination of the residual plot as shown in Figure 4 indicated no systematic patterns were left in the model, and the result of the Shapiro-Wilk test also showed that the residual revealed normal distribution with $\mathrm{W}=0.989$ and $\operatorname{Pr}<\mathrm{W}=0.42$.

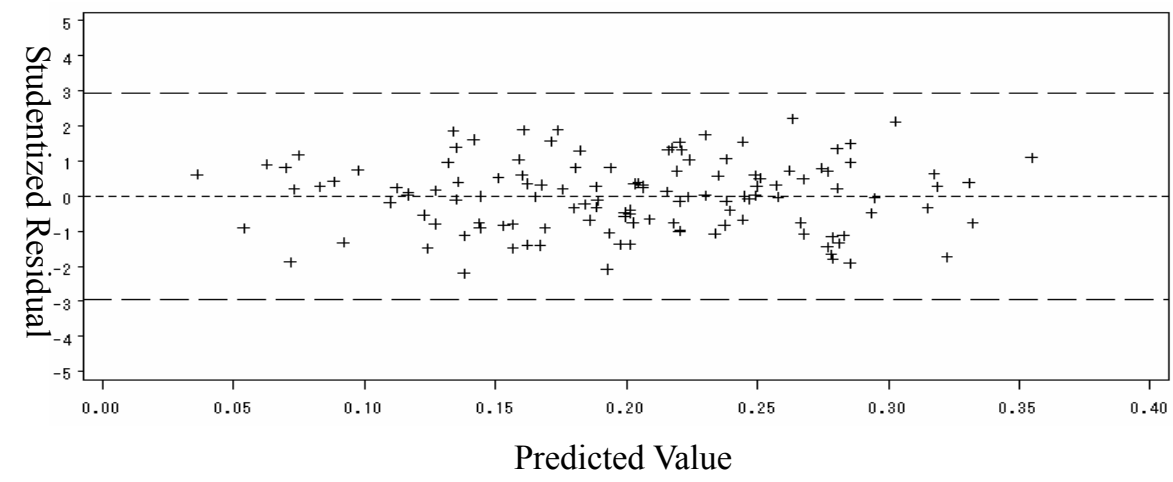

Figure 4. Residual Plot of the Best-fit Model

The true out-of-sample forecast accuracy was measured to test the prediction capability of the best-fit model. For this, the lane-change samples were divided into an initialization set and a test set, where the initialization set was used for model estimation and forecast was made for the test set. The initialization set contained $70 \%$ of samples randomly selected from the lane-change sampling data and the rest constituted the test set. A total of five times of tests were made, and the prediction accuracy was measured by the mean absolute percentage error, which revealed an average of $4.5 \%$. Furthermore, the use of $95 \%$ and $90 \%$ prediction intervals as threshold boundaries to evaluate forecast capability of the proposed models and the results showed an average of 1.4 and 3.2 of true values falling outside the boundaries, respectively.

\section{DISCUSSION AND CONCLUSIONS}

Results of two-way ANOVA demonstrated that there was a significant lane-change direction $\mathrm{x}$ vehicle speed in the target lane interaction, and further analyses showed that there was a simple effect for vehicle speed in the target lane for the left-to-right group of the type of lane-change direction factor. The interaction effect shows that both factors are critical to the value of the accepted gap of lane changes, which also echoes the findings in literature. This simple effect implies that left-to-right lane-change maneuvers are more difficult for bus drivers compared to right-to-left lane change maneuvers. The reason for the difference could be the effect of the size of a bus, for which it may be more difficult for drivers to measure accurately the size of a gap and 
judge the potential hazards. However, further investigation with more samples or different driving scenes might be necessary to ensure the significance of the effect.

The result of the forecasting model indicated that the subject vehicle's speed, vehicle speed in the target lane and longitudinal spacing between the subject vehicle and its lead and lag vehicles in the target lane at the moment of the discretionary lane change are critical factors affecting bus drivers' ability to make a successful discretionary lane change maneuver. Both this finding and that of ANOVA confirmed the results of literature showing that the speeds of the subject vehicle and vehicles in the target lane were crucial factors affecting the value of an accepted gap for lane changes. The test results for the prediction model shown in the previous section also demonstrated a promising potential for application in the development of lane-change support systems. However, further investigation and validation of this model with more samples and other scenarios like the curvature section of roadway, as well as how to incorporate this model into the design of the prediction module of a lane-change support system, are all necessary to ensure its practical applicability.

\section{ACKNOWLEDGMENTS}

The financial support from Taiwan National Science Councils with grant number NSC952211E216054 is gratefully acknowledged.

\section{REFERENCES}

Ahmed, K.I., Ben-Akiva, M.E., Koutsopoulos, H.N., \& Mishalani, R.G. (1996). Models of freeway lane changing and gap acceptance behavior. In J. Lesort (Ed.), Transportation and Traffic Theory. Pergamon, 501-515.

FHWA. (1998). CORSIM User Manual (1.04 ed.). McLean, Virginia: US DOT, Office of Safety and Traffic Operations R\&D, Intelligent Systems and Technology Division (HSR-10),

Gipps, P.G. (1986). A model for the structure of lane-changing decisions. Transportation Research, 20B(5), 403-414.

Jula, H., Kosmatopoulos, E B., \& Ioannou, P.A. (2000). Collision avoidance analysis for lane changing and merging. IEEE Transactions on Vehicular Technology, 49(6), 2295-2308.

NHTSA. (1994). Examination of lane change crashes and potential IVHS countermeasures (DOT HS 808 071). Washington, DC: U.S. Government Printing Office.

-. (2004). A comprehensive examination of naturalistic lane-changes (DTNH22-00-C-07007, Task Order 4). Washington, DC: U.S. Government Printing Office.

Mahmassani, H., \& Sheffi, Y. (1981). Using gap sequences to estimate gap acceptance functions. Transportation Research, 15B, 143-148.

Toledo, T., Koutsopoulos, H.N., \& Ben-Akiva, M E. (2003). Modeling integrated lane-changing behavior. Transportation Research Board, 74th Annual Meeting CD-ROM.

Winsum, W. van, Waard, D. de, \& Brookhuis, K.A. (1999). Lane change maneuvers and safety margins. Transportation Research, $2 F, 139-149$. 